\title{
The Devil in the Details: What Everybody Should Know When Running DFT Calculations
}

Pierpaolo Morgante, Roberto Peverati*

Chemistry Program, Florida Institute of Technology, 150 W University Blvd., Melbourne, Florida, 32901, USA.

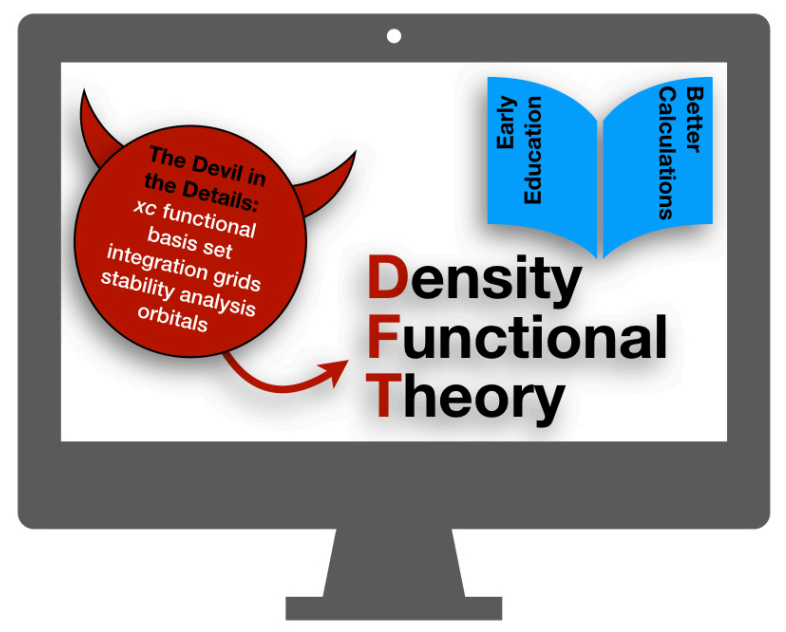

\begin{abstract}
Density functional theory (DFT) has rapidly became the method of choice for the calculation of the electronic structure of atoms and molecules in both the teaching and the research environments. Despite a long history of successes in both fields, DFT still retains several limitations due to the fact that it relies on approximations. Advances on the research side have recently contributed to understand and reduce several of these limitations. The most important results have been obtained in the last two decades by using large databases of accurate data. These databases provided a better understanding of the limitations of current approximations and facilitated the development of more accurate ones. The educational literature, on the other side, has unfortunately remained behind. It is still common, in fact, to find computational laboratory courses that use outdated approximations, without providing a clear report of their limitations, and also failing to mention the modern alternatives. This article introduces a laboratory notebook for a computational chemistry course based on recent advances in DFT research. New approximations and neglected techniques are presented and discussed using data from modern research databases. The course is aimed at upper undergraduate and graduate students, but it includes enough research examples to be interesting for occasional practitioners and beginner researchers as well.
\end{abstract}

\section{INTRODUCTION}

Computational chemistry has benefitted from the increase in power and performance of computers in the past decades, and improvements have been so fast that it is now common to find undergraduate and graduate laboratories ${ }^{1-36}$ where calculations can be run on simple desktop computers, or even on laptops. The main goal of these activities is to introduce students to the common practices in the field of computational chemistry. Many laboratories include geometry optimizations and frequency calculations at different levels of theory, specifically semiempirical ${ }^{37}$ or Hartree-Fock (HF) methods, 38,39 as well as density functional theory (DFT). ${ }^{40}$ There is no doubt that these activities are important in education, but most of them unfortunately suffer of at least one of two major drawbacks: 1) they are either too introductory in nature, downplaying the difficulties of performing calculations in a research environment, or 2) they do not reflect the most recent advances in the research community. A survey of the current educational literature 
reveals how introductory material presents calculation in a simplified manner (for example by using simple graphical interfaces and outdated methods $^{1,2,4-6,8,13,18,21,23,28,41}$ ), devaluing the role of computational chemistry in chemical research, while more advanced material that uses DFT still fails to suggest approximations developed in the last twenty years $2,3,7,9-11,14-18,21,22,24-27,29-36,42-44$, neglecting two decades of significant research advances.

Introductory laboratory courses in other fields of chemistry usually adopt a more cautious approach. ${ }^{45-47}$ For example, in the analytical chemistry laboratory, 48 the challenge of manual titrations is often initially preferred to more modern automatic techniques. They are conceptually simple experiments, but they are complicated by the usage of manual equipment (often obsolete in modern research laboratories), so that the students can realize the importance of being attentive in the laboratory environment, as well as the struggles to get reliable and accurate experimental data. In most advanced courses, however, modern instrumentation are introduced and used, ${ }^{49-51}$ so that the advanced students are aware of their strengths and limitations in the research setting. On the other hand, in the introductory computational chemistry laboratories many efforts are spent to make the field look more appealing than it is in reality. In many introductory computational laboratories, students learn basic commands to run calculations on molecules that are usually small and uncomplicated. Moreover, most courses are specifically designed to be used with a specific program, usually Spartan $^{52}$ or Gaussian. ${ }^{53}$ Unfortunately, within these simple (mostly graphical) environments, students learn how to perform calculations "the easy way", instead of learning how to be aware of the difficulties associated with a computational experiment. Advanced courses do not rectify this misconception either, spreading the wrong message that "if you own a computer, you can run a quantum chemical calculation". This does not reflect the day-to-day life of a researcher in the field. Computational chemists often struggle in their research between different software, complicated command line interfaces, and wildly discordant results that do not match experiments.

While DFT is becoming ubiquitous throughout the entire chemistry literature, several aspects of its modern research are often neglected by the educational side as well. For example, the essential developments in the approximations to the exchange-correlation functional (from here on, simply abbreviated as $x c$ functionals) that happened in the last two decades, are usually completely neglected by most educational courses. Several of these new advances have been facilitated by the development of large databases of accurate data of broad chemical interest. 54-59 For example, topics at the center of the last decades of DFT research, such as dispersion corrections, ${ }^{60-65}$ broadly applicable meta-GGA functionals, $55,56,66-68$ and functionals for strong correlation, $54,55,69-74$ are not even presented to students until their post-educational development. Introducing such important topics to DFT students early on in their educational program has the benefit of reducing a potentially dangerous oversight. It is important to stress out at this stage, that it is not our objective to disregard reliable older approximations and laboratory experiences, nor to provide answers on how to solve all the issues that might arise in DFT calculations. For several simple cases, in fact, thirty or forty years old $x c$ functionals-such as the popular B3LYP approximation ${ }^{75-78-a r e}$ perfectly capable of providing accurate answers. However, it is our purpose to show that these older $x c$ functionals sometimes fail to provide reliable results, and newer and more advanced methods are indeed available. Moreover, for relatively unknown systems, we simply cannot predict in advance whether older $x c$ functionals are going to be reliable or not. Another goal is to introduce some of these issues relatively early in the students computational training, so we can educate new practitioners that are better informed on the challenges of computational chemistry. A new generation of knowledgeable computational scientists will promote the development of new ideas and the search for expert advice when complicated cases emerge. Using the analogy to the introductory wet laboratory courses once again, often the overall pedagogical message of such courses is not that everybody can do complex chemical experiments, but rather to provide a tool to judge situations when it is necessary to seek the guidance of an expert. Our aim with this work is to replicate this strategy for computational laboratories as well.

\section{PEDAGOGICAL APPROACH AND OBJECTIVES}

In order to bridge the gap between developers and users of computational chemistry, we present here a new set of computational experiments that are coming directly from DFT research. All of the data for the experiments are taken from the 
ACCDB collection of modern databases, 59 and originally come from the GMTKN55,58 MGCDB84,57 or Minnesota 201554-56 databases. The experiments are selected by keeping in mind students and educators who are interested in learning and teaching computational chemistry from a more cautious point of view. All calculations require a software that can handle Kohn-Sham density functional theory (KS-DFT) calculations. They can be run on either commercial or open-source quantum chemistry programs, as long as the software includes the most common $x c$ functional approximations. The choice of whether to use a graphical user interface (GUI) or not, is left to the instructor. In general, we tested all the calculations with Gaussian ${ }^{53}$ and Q-Chem ${ }^{79}$ using their corresponding command line interfaces. We have also successfully used the IQmol80 GUI to plot the orbitals, but the experiments are simple enough to be easily ported to any other GUI. Regardless of the choice of software, all calculations are small enough to run on a desktop or a laptop computer, but the experiments are challenging enough to make the outcomes interesting to discuss. The main pedagogical objective of the experiments is to deliver a better understanding of what questions computational chemistry (DFT in particular) can, or cannot, answer.

The delivery mechanism that we chose is a laboratory notebook composed of 14 weekly experiments that can be used as a guide to complement a one-semester (14-16 weeks) computational chemistry course on density functional theory for advanced undergraduate or graduate students. The notebook has been successfully tested in the Special Topics in Physical Chemistry course taught by the Corresponding Author since 2016 in the Fall Semester of the Chemistry Program at Florida Tech. This course is dual listed for upper level (fourth year) undergraduate and graduate students, and it is specifically focused on practical aspects of DFT calculations. Within the Florida Tech Chemistry curriculum, the Special Topics course follows the Advanced Physical Chemistry course, which is taught also by the Corresponding Author during the preceding Spring Semester. The theoretical foundation of density functional theory is established in the Advanced Physical Chemistry course and predates the usage of this notebook. Therefore, we do not recommend using this notebook alone as a first introduction to computational chemistry, unless it is accompanied by a theory class where the foundation is established. The theory class can either happen in the prior semester-as is the case at Florida Tech-or concurrently. For this reason, there is no experiment specifically focused on the explanation of the theoretical foundations, but rather they are assumed as already established. Examples of pre-requisite notions are: the nature of the many approximations to the $x c$ functional, the definition of a basis set, the construction of a real-space integration grid, and charge and orbital-localization techniques.

\section{STRUCTURE OF THE NOTEBOOK}

This laboratory notebook is intended mainly as a guide for beginners or non-experts in the field, however, some of the experiments can be useful for experts in a research environment as well, since they highlight several overlooked aspects of DFT. The structure of the notebook is summarized in Table $\mathbf{1}$, including the corresponding learning objective for each experiment. The notebook begins with an introductory experience (Experiment 0), where the students are introduced to computational databases, the basic concepts on how to classify $x c$ functionals, ${ }^{81}$ and the typical associated jungle of acronyms. As a suggestion, the students are also referred to read at least one of the modern reviews on DFT57,82-86 before starting the laboratory. The rest of the notebook is divided into two parts: the first part deals with common pitfalls in the choice of the method for a DFT calculation (experiment 1-7), while the second part delves deeper into neglected details and misused techniques (experiment 8-14).

Notebook Part 1 (1-7): Pitfalls in the choice of the method

The term "method" is used throughout the notebook to indicate a specific combination of $x c$ functional and basis set. The first three experiments include organic and inorganic reactions to demonstrate that selecting an $x c$ functional is not necessarily an easy task. Even systems that might appear simple from a chemical perspective-like calculating the rotational barrier of ethane or the difference in energies of a DielsAlder reaction-can be challenging for some $x c$ functional. Each experiment deals with a set of reactions that can be classified as easy 
Table 1. Structure of the laboratory notebook and corresponding learning objective.

\begin{tabular}{|c|c|c|}
\hline Week: & Experiment: & Learning Objective: \\
\hline 0 & A Note on Choosing a Good Functional & Classify $x c$ functionals \\
\hline \multicolumn{3}{|c|}{ Part 1: Pitfalls in the choice of the method } \\
\hline 1 & Applying DFT to Organic Chemistry - Part A & \\
\hline 2 & Applying DFT to Organic Chemistry - Part B & Common traps in the selection of $x c$ functionals \\
\hline 3 & Difficult Cases for DFT & \\
\hline 4 & Basis Set Incompleteness Error & \\
\hline 5 & Basis Set Superposition Error & Common traps in the selection of basis sets \\
\hline 6 & Review of Modern Basis Sets & \\
\hline 7 & $\begin{array}{l}\text { Why is the B3LYP/6-31G* Level of Theory } \\
\text { So Successful? }\end{array}$ & Limitations of B3LYP $/ 6-31 \mathrm{G}^{*}$ and error cancellation \\
\hline
\end{tabular}

Part 2: Neglected details and misused techniques

\begin{tabular}{cll}
8 & Integration Grids $1:$ The Argon Dimer & Integration grids \\
9 & Integration Grids 2: The Case of But-2-yne & \\
\hline 10 & Stability Analysis and Transition Metals & Stability analysis \\
\hline 11 & Analysis of Atomic Charges & Different flavors of atomic charges \\
\hline 12 & Localized and Delocalized Orbitals & Orbitals meaning and usage \\
\hline 13 & Comparison with Experimental Results & $\begin{array}{l}\text { Required corrections to compare with experimental } \\
\text { values }\end{array}$ \\
\hline 14 & Evaluation/Test: Collaborative Project & $\begin{array}{l}\text { Setting up and running simple calculations, but } \\
\text { knowing when to seek for an expert }\end{array}$ \\
\hline
\end{tabular}

(Experiment 1), medium (Experiment 2), and hard (Experiment 3). Particular emphasis is put on the fact that there is no recipe to determine in advance to which class a specific chemical problem belongs to, hence no strategy for predicting the performance of an $x c$ functional a priori. A method that is perfectly suitable for one specific chemical problem (such as studying the rotational barrier in ethane in Experiment 1), can be uncapable of providing reasonable results for a similarly complicated system (such as the Diels-Alder reaction in Experiment 2). Experiment 3 includes reactions that are notoriously difficult for most $x c$ functionals and is intended as a warning for students to seek expert advice in cases where different methods provide wildly discordant results.

The following three experiments use a similar approach to discuss about basis sets. In the first two experiments the two main errors associated with the selection of a necessarily truncated basis set are presented and analyzed: the basis set incompleteness error (BSIE, Experiment 4), 87-89 and the basis set superposition error (BSSE, Experiment 5). ${ }^{90}$ In Experiment 6 students will learn how basis sets of triple- $\zeta$ quality are necessary to obtain accurate results for several energies, and will analyze the adequacy of different families of basis sets for DFT calculations. The Pople family, ${ }^{91-98}$ for example, is the most used basis set in the education literature. However, a recent study demonstrates that it is not optimal for DFT.99 Similarly, the Dunning family ${ }^{100-103}$ has been optimized specifically for correlated wave function methods, but its members usually contain an unnecessarily large number of basis functions for DFT calculations. 104 For these reasons, we suggest moving away from Pople's and Dunning's basis sets in favor of basis sets that are specifically optimized for DFT-like the ones from Ahrlichs ${ }^{105}$ or Jensen 106-109 - which are the basis set of choice for all the remaining calculations in the notebook.

The first half of the notebook concludes with an experiment specifically designed to show the limitations of the popular B3LYP/6-31G* method. Despite many documented failures in various areas of chemistry, ${ }^{110-114}$ the B3LYP ${ }^{75-78}$ hybrid functional used in combination with the $6-31 G^{*}$ basis set is the de facto standard for calculations involving organic molecules, especially for teaching purposes. While B3LYP/6-31G* is perfectly fine for some simple system, many laboratory notebooks $18,21,22,24,44,115$ use it even for 
reactions that are known to be problematic for this functional and basis set. In Experiment 7, the students will review the calculations from experiments 1, 2, and 3 using B3LYP and the 6$31 \mathrm{G}^{*}$ basis set to highlight successes and failures. We believe it is time to train a new generation of chemists to be aware of the limitations of B3LYP/6-31G*, and to seek better methods for problematic cases.

Notebook Part 2 (8-14): Neglected details and misused techniques

The second portion of the notebook is dedicated to details of DFT calculations and accessory techniques that are often overlooked by occasional practitioners. Experiment 8 and 9 explore the limitation of the integration grids. In Experiment 8, students understand that the choice of an integration grid can have a significant impact on the quality of electronic energies, as shown for some members of the Minnesota family of functionals, for which a grid of at least 99 radial and 590 angular points is necessary to converge some problematic cases, ${ }^{116-118}$ Often such grids are not the default that is automatically chosen by quantum chemistry program, but their usage worsen the cost of a calculation only moderately, and they should be preferred over the default parameters in most cases. Experiment 9 is instead focused on the effects of integration grids on the calculation of thermodynamic properties, such as entropies, Gibbs' free energies and vibrational frequencies. ${ }^{119}$

Experiment 10 is dedicated to the stability analysis of the final self-consistent-field (SCF) solution. For systems with almost-degenerate lowlying states, the SCF procedure might in fact converge to a state that is not the lowest in energy. This higher energy SCF solution is said to be unstable, while only the lowest energy SCF solution for each spin multiplicity will be stable. Most modern quantum chemistry software includes algorithms to check the stability of the final solution, however they are never included in the default settings, and they are too often forgotten, even by experienced users. While in principle every calculation-even on simple "highgap" cases-can erroneously converge to an unstable SCF solution, it becomes crucial to perform stability analysis for systems that have several low-lying states, such as transition metals. ${ }^{120,121}$ After this experiment, students should be aware of this procedure and will hopefully use it in most cases, especially in case of doubts such as transition metals and other potentially "low-gap" systems.

Experiments 11 and 12 deal with two concepts that are used (and sometimes abused) in several field of chemistry: atomic partial charges and molecular orbitals. For example, in organic chemistry the partial charge of an atom is determined after drawing the most meaningful Lewis structure by taking into account valence electrons, electrons shared in a bond, and lone pairs. In computational chemistry, there are many different ways to calculate atomic charges, ${ }^{122}$ and the students will use the Mulliken ${ }^{123-126}$ and the ChelPG ${ }^{127,128}$ charges in Experiment 11. This experiment is designed to show the limitations of these population analysis techniques, and to alert students that the results might vary wildly depending on the chosen technique and method. Another concept that is broadly used in chemistry is the concept of molecular orbitals. An orbital is a single-particle wave function that is used to describe the behavior of one "electron" (or a "pair of electrons") in an atom or a molecule. A quotation mark around electron is necessary at this point to emphasize the disconnect between the concept of "electron" as used by chemists and its definition as used by physicists. ${ }^{129}$ Because of their mathematical definition, orbitals can describe the physicist's electron only in one-electron systems. In a many-electron system, electrons are in principle correlated (i.e. they interact with each other), and because of this interaction, it is not possible to single out the behavior of one of them and describe it with a single-particle wave function. However, it is possible to approximate it by constructing a quasi-particle (which chemists named "electron"), described by a single-particle wave function (orbital) that includes the average interaction with all other similar quasi-particles via a dressed term. While this important distinction is wellknown in the theoretical chemistry community, ${ }^{129-131}$ it is unfortunately too often overlooked by computational practitioners. For this reason, it is important to stress out that orbitals-in particular molecular orbitals-are not real entities, and they certainly cannot be measured. ${ }^{130-132}$ The molecular orbitals resulting from a calculation are called canonical orbitals. The canonical orbitals in a DFT calculation are built as linear combination of atomic basis functions, and the associated coefficients obtained from a KS-DFT calculation are those that provide the best density at the end of the SCF procedure. However, such coefficients are 
invariant to unitary transformations, and consequently they can be localized using different localization schemes. ${ }^{133-136}$ In Experiment 12, the Boys localization scheme ${ }^{133}$ is chosen to show that the shapes of the canonical orbitals do not carry any special meaning, 131 because they can be manipulated by simple unitary transformations. Similarly, the unoccupied orbitals of molecules are obtained from the symmetry constraint dictated by the occupied orbitals, and they are never optimized in the SCF procedure. For this reason, their shapes have even less physical meaning, and in the case of KS-DFT calculations, even their energies become questionable. $1, \underline{2}$

Finally, Experiments 13 and 14 show how to use DFT calculations to compare results with experimental data. Experiment 13 shows how calculating the dissociation energy of a bond, while conceptually a simple task, might result in several unexpected computational issues. Specifically, the homolytic dissociation of a single bond yields two radical species, which are likely to require a multi-determinant approach, ${ }^{137}$ and many $x c$ functionals might struggle with them. Students will learn how the calculated total atomization energies of small molecules cannot be directly compared to the respective enthalpies of formation. It is important that the students realize that this is not a limitation of the computational methods themselves, but rather a byproduct of the fact that the computational setup is different than the experimental one. Several corrections that account for such differences must be applied to the calculated data in order for a meaningful comparison. Experiment 14, which is the last experiment in the notebook, is designed as a test for the evaluation of the students' performance in the entire laboratory course. It is constructed so it can be run as a collaborative project, but it can also be simply converted to an individual test. The task for the students is to perform a complete computational study using the concepts that they learned from the previous experiments. The system under investigation is challenging, but it does not differ significantly from other problems that the students faced in the previous experiments. A passing grade is achieved if the students use a modern $x c$ functional with dispersion corrections and a triple- $\zeta$ basis set as a good compromise between accuracy and calculation time. In addition, hopefully the $(99,590)$ integration grid is used, and since the system includes transition metals, also stability analysis.

\section{CONCLUSIONS}

We presented a new notebook for a semesterlong computational chemistry laboratory course containing 14 weekly experiments. This notebook can be integrated with a theory course on density functional theory for advanced undergraduate or graduate students. DFT is becoming the standard method for quantum chemical calculations in both the teaching and research environments. We designed the experiments to demystify important techniques that are too often forgotten by students and occasional practitioners of computational chemistry. All the experiments come from research databases, and they deal with systems of broad chemical interest. To our knowledge, this is the first example of usage of modern computational research databases in an educational environment.

While this notebook is designed mainly as a teaching tool, the selection of real research examples from modern computational databases extends its value well beyond the classroom. Its main strength in the research environment is in line with our initial goal of highlighting what everybody should know when running a DFT calculation. This pedagogical goal is achieved by bringing awareness to the common pitfalls, neglected details, and misused techniques that computational scientists must be aware of when performing DFT calculations.

\section{AUTHOR INFORMATION}

\section{Corresponding Author}

*E-mail: rpeverati@fit.edu

\section{REFERENCES}

(1) Ramos, M. J.; Fernandes, P. A.; Melo, A. Modeling Chemical and Biological Systems: A Successful Course for Undergraduate Students. J. Chem. Educ. 2004, 81 (1), 72.

(2) Feller, S. E.; Dallinger, R. F.; McKinney, P. C. A Program of Computational Chemistry Exercises for the First-Semester General Chemistry Course. J. Chem. Educ. 2004, 81 (2), 283.

(3) Halpern, A. M. Computational Studies of Chemical Reactions: The HNC-HCN and CH3NC-CH3CN Isomerizations. J. Chem. Educ. 2006, 83 (1), 69.

(4) Montgomery, C. D. [Pi] $\pi$ Backbonding in Carbonyl Complexes and Carbon-Oxygen Stretching Frequencies: A Molecular Modeling Exercise. J. Chem. Educ. 2007, 84 (1), 102. 
(5) Glish, L.; Hanks, T. W. Computational Analysis of Stereospecificity in the Cope Rearrangement. J. Chem. Educ. 2007, 84 (12), 2001.

(6) Cramer, C. J.; Bumpus, J. A.; Lewis, A.; Stotts, C. Characterization of High Explosives and Other Energetic Compounds by Computational Chemistry and Molecular Modeling. J. Chem. Educ. 2007, 84 (2), 329.

(7) Halpern, A. M.; Ramachandran, B. R.; Glendening, E. D. The Inversion Potential of Ammonia: An Intrinsic Reaction Coordinate Calculation for Student Investigation. $J$. Chem. Educ. 2007, 84 (6), 1067.

(8) Patalinghug, W. C.; Chang, M.; Solis, J. Predicting the Shifts of Absorption Maxima of Azulene Derivatives Using Molecular Modeling and ZINDO CI Calculations of UVVis Spectra. J. Chem. Educ. 2007, 84 (12), 1945.

(9) Rowley, C. N.; Woo, T. K.; Mosey, N. J. A Computational Experiment of the Endo versus Exo Preference in a Diels-Alder Reaction. J. Chem. Educ. 2009, 86 (2), 199.

(10) Clauss, A. D.; Nelsen, S. F. Integrating Computational Molecular Modeling into the Undergraduate Organic Chemistry Curriculum. J. Chem. Educ. 2009, 86 (8), 955.

(11) Halpern, A. M. Structural and Thermodynamic Properties of the Argon Dimer: A Computational Chemistry Exercise in Quantum and Statistical Mechanics. J. Chem. Educ. 2010, 87 (2), 174-179.

(12) Halpern, A. M. Dispersion Interactions between Rare Gas Atoms: Testing the London Equation Using $\mathrm{Ab}$ Initio Methods. J. Chem. Educ. 2011, 88 (2), 174-177.

(13) McNaught, I. J. Testing and Extending VSEPR with WebMO and MOPAC or GAMESS. J. Chem. Educ. 2011, 88 (4), 421425.

(14) Johnson, L. E.; Engel, T. Integrating Computational Chemistry into the Physical Chemistry Curriculum. J. Chem. Educ. 2011, 88 (5), 569-573.

(15) Linenberger, K. J.; Cole, R. S.; Sarkar, S. Looking Beyond Lewis Structures: A General Chemistry Molecular Modeling Experiment Focusing on Physical Properties and Geometry. J. Chem. Educ. 2011, 88 (7), 962965.

(16) Marzzacco, C. J.; Baum, J. C. Computational Chemistry Studies on the
Carbene Hydroxymethylene. J. Chem. Educ. 2011, 88 (12), 1667-1671.

(17) Glendening, E. D.; Halpern, A. M. A Computational Study of Rare Gas Clusters: Stepping Stones to the Solid State. J. Chem. Educ. 2012, 89 (12), 1515-1521.

(18) Wang, L. Using Molecular Modeling in Teaching Group Theory Analysis of the Infrared Spectra of Organometallic Compounds. J. Chem. Educ. 2012, 89 (3), 360-364.

(19) Montgomery, C. D. Factors Affecting Energy Barriers for Pyramidal Inversion in Amines and Phosphines: A Computational Chemistry Lab Exercise. J. Chem. Educ. 2013, 90 (5), 661-664.

(20) Csizmar, C. M.; Daniels, J. P.; Davis, L. E.; Hoovis, T. P.; Hammond, K. A.; McDougal, O. M.; Warner, D. L. Modeling $\mathrm{S}_{\mathrm{N}} 2$ and E2 Reaction Pathways and Other Computational Exercises in the Undergraduate Organic Chemistry Laboratory. J. Chem. Educ. 2013, 90 (9), 1235-1238.

(21) Montgomery, C. D. A Mechanistic Study of the Migratory Insertion Reaction: A Computational Chemistry Exercise. $J$. Chem. Educ. 2013, 90 (10), 1396-1400.

(22) Nassabeh, N.; Tran, M.; Fleming, P. E. Dissociation of the Ethyl Radical: An Exercise in Computational Chemistry. $J$. Chem. Educ. 2014, 91 (8), 1248-1253.

(23) Albrecht, B. Computational Chemistry in the Undergraduate Laboratory: A Mechanistic Study of the Wittig Reaction. J. Chem. Educ. 2014, 91 (12), 2182-2185.

(24) Ugone, V.; Garribba, E.; Micera, G.; Sanna, D. Equilibrium between Different Coordination Geometries in Oxidovanadium(IV) Complexes. J. Chem. Educ. 2015, 92 (6), 1098-1102.

(25) Martini, S. R.; Hartzell, C. J. Integrating Computational Chemistry into a Course in Classical Thermodynamics. J. Chem. Educ. 2015, 92 (7), 1201-1203.

(26) Garino, C.; Terenzi, A.; Barone, G.; Salassa, L. Teaching Inorganic Photophysics and Photochemistry with Three Ruthenium(II) Polypyridyl Complexes: A Computer-Based Exercise. J. Chem. Educ. 2016, 93 (2), 292298.

(27) Esselman, B. J.; Hill, N. J. Integration of Computational Chemistry into the Undergraduate Organic Chemistry 
Laboratory Curriculum. J. Chem. Educ. 2016, 93 (5), 932-936.

(28) Best, K. T.; Li, D.; Helms, E. D. Molecular Modeling of an Electrophilic Addition Reaction with "Unexpected" Regiochemistry. J. Chem. Educ. 2017, 94 (7), 936-940.

(29) Schuster, M. L.; Peterson, K. P.; Stoffregen, S. A. Isobutylene Dimerization: A DiscoveryBased Exploration of Mechanism and Regioselectivity by NMR Spectroscopy and Molecular Modeling. J. Chem. Educ. 2018, 95 (6), 1040-1044.

(30) Adams, W.; Sonntag, M. D. Vibrational Spectroscopy of Hexynes: A Combined Experimental and Computational Laboratory Experiment. J. Chem. Educ. 2018, 95 (7), 1205-1210.

(31) Rodríguez González, M. C.; Hernández Creus, A.; Carro, P. Combining Electrochemistry and Computational Chemistry to Understand Aryl-Radical Formation in Electrografting Processes. J. Chem. Educ. 2018, 95 (8), 1386-1391.

(32) Pemberton, A. T.; Magers, D. B.; King, D. A. Integrated TGA, FTIR, and Computational Laboratory Experiment. J. Chem. Educ. 2019, 96 (1), 132-136.

(33) Mazzuca, J. W.; Downing, A. R.; Potter, C. Empirically Corrected Electronic Structure Calculations Applied to the Enthalpy of Combustion Physical Chemistry Laboratory. J. Chem. Educ. 2019, 96 (6), 1165-1170.

(34) Thu Le, N. N.; Klinkerch, E. J.; Thamattoor, D. M. Stereochemistry of Dichlorocarbene Addition to Alkenes: A Collaborative, Discovery-Based Experiment for the Organic Chemistry Laboratory. J. Chem. Educ. 2019, 96 (8), 1727-1730.

(35) Rodríguez Ortega, P. G.; Montejo, M.; Valera, M. S.; López González, J. J. Studying the Effect of Temperature on the Formation of Hydrogen Bond Dimers: A FTIR and Computational Chemistry Lab for Undergraduate Students. J. Chem. Educ. 2019, 96 (8), 1760-1766.

(36) Miller, D. P.; Phillips, A.; Ludowieg, H.; Swihart, S.; Autschbach, J.; Zurek, E. The Computational Design of Two-Dimensional Materials. J. Chem. Educ. 2019, 96 (10), 2308-2314.

(37) Thiel, W. Semiempirical Quantum-Chemical Methods. Wiley Interdiscip. Rev. Comput. Mol. Sci. 2013, 4 (2), 145-157.

(38) Hartree, D. R. The Wave Mechanics of an Atom with a Non-Coulomb Central Field.
Part I. Theory and Methods. Math. Proc. Camb. Philos. Soc. 1928, 24 (1), 89-110.

(39) Fock, V. Näherungsmethode zur Lösung des quantenmechanischen

Mehrkörperproblems. Z. Für Phys. 1930, 61 (1-2), 126-148.

(40) Kohn, W.; Sham, L. Self-Consistent Equations Including Exchange and Correlation Effects. Phys. Rev. 1965, 140 (4A), A1133-A1138.

(41) Simeon, T.; Aikens, C. M.; Tejerina, B.; Schatz, G. C. Northwestern University Initiative for Teaching NanoSciences (NUITNS): An Approach for Teaching Computational Chemistry to Engineering Undergraduate Students. J. Chem. Educ. 2011, 88 (8), 1079-1084.

(42) Martin, C. B.; Vandehoef, C.; Cook, A. The Use of Molecular Modeling as "Pseudoexperimental" Data for Teaching VSEPR as a Hands-On General Chemistry Activity. J. Chem. Educ. 2015, 92 (8), 13641368.

(43) Altarawneh, M.; Dlugogorski, B. Z. Introducing Quantum Chemistry in Chemical Engineering Curriculum. J. Chem. Educ. 2018, 95 (9), 1562-1571.

(44) Hill, N. J.; Bowman, M. D.; Esselman, B. J.; Byron, S. D.; Kreitinger, J.; Leadbeater, N. E. Ligand-Free Suzuki-Miyaura Coupling Reactions Using an Inexpensive Aqueous Palladium Source: A Synthetic and Computational Exercise for the Undergraduate Organic Chemistry Laboratory. J. Chem. Educ. 2014, 91 (7), 1054-1057.

(45) Timberlake, K. C. Laboratory Manual for General, Organic, and Biological Chemistry; Pearson Education: Upper Saddle River (N.J.), 2014.

(46) Tro, N. J.; Vincent, J. B.; Livingston, E. Laboratory Manual for Chemistry: A Molecular Approach, Fourth Edition.; Pearson: Boston, 2017.

(47) Canal, J. P.; Lowe, J.; Fong, R. Improving Students' Practical Laboratory Techniques through Focused Instruction and Assessment. In ACS Symposium Series; Schultz, M., Schmid, S., Holme, T., Eds.; American Chemical Society: Washington, DC, 2016; Vol. 1235, pp 137-157.

(48) Sheppard, K. High School Students' Understanding of Titrations and Related Acid-Base Phenomena. Chem Educ Res Pr. 2006, 7 (1), 32-45. 
(49) Bandyopadhyay, S.; Rathod, B. B. The Sound and Feel of Titrations: A Smartphone Aid for Color-Blind and Visually Impaired Students. J. Chem. Educ. 2017, 94 (7), 946949.

(50) Tee, N. Y. K.; Gan, H. S.; Li, J.; Cheong, B. H.-P.; Tan, H. Y.; Liew, O. W.; Ng, T. W. Developing and Demonstrating an Augmented Reality Colorimetric Titration Tool. J. Chem. Educ. 2018, 95 (3), 393-399.

(51) Soong, R.; Agmata, K.; Doyle, T.; Jenne, A.; Adamo, A.; Simpson, A. J. Rethinking a Timeless Titration Experimental Setup through Automation and Open-Source Robotic Technology: Making Titration Accessible for Students of All Abilities. J. Chem. Educ. 2019, 96 (7), 1497-1501.

(52) Spartan'18; Spartan Inc: Irvine, CA, USA, 2019.

(53) Frisch, M. J.; Trucks, G. W.; Schlegel, H. B.; Scuseria, G. E.; Robb, M. A.; Cheeseman, J. R.; Scalmani, G.; Barone, V.; Petersson, G. A.; Nakatsuji, H.; et al. Gaussian $16 \mathrm{Rev}$. C.01; Gaussian Inc.: Wallingford, CT, 2016.

(54) Yu, H. S.; Zhang, W.; Verma, P.; He, X.; Truhlar, D. G. Nonseparable ExchangeCorrelation Functional for Molecules, Including Homogeneous Catalysis Involving Transition Metals. Phys. Chem. Chem. Phys. 2015, 17 (18), 12146-12160.

(55) Yu, H. S.; He, X.; Truhlar, D. G. MN15-L: A New Local Exchange-Correlation Functional for Kohn-Sham Density Functional Theory with Broad Accuracy for Atoms, Molecules, and Solids. J. Chem. Theory Comput. 2016, 12 (3), 1280-1293.

(56) Yu, H. S.; He, X.; Li, S. L.; Truhlar, D. G. MN15: A Kohn-Sham Global-Hybrid Exchange-Correlation Density Functional with Broad Accuracy for Multi-Reference and Single-Reference Systems and Noncovalent Interactions. Chem. Sci. 2016, 7 (8), 5032-5051.

(57) Mardirossian, N.; Head-Gordon, M. Thirty Years of Density Functional Theory in Computational Chemistry: An Overview and Extensive Assessment of 200 Density Functionals. Mol. Phys. 2017, 115 (19), 2315-2372.

(58) Goerigk, L.; Hansen, A.; Bauer, C.; Ehrlich, S.; Najibi, A.; Grimme, S. A Look at the Density Functional Theory Zoo with the Advanced GMTKN55 Database for General Main Group Thermochemistry, Kinetics and
Noncovalent Interactions. Phys. Chem. Chem. Phys. 2017, 19 (48), 32184-32215.

(59) Morgante, P.; Peverati, R. ACCDB: A Collection of Chemistry Databases for Broad Computational Purposes. J. Comput. Chem. 2019, 40, 839-848.

(60) Becke, A. D.; Johnson, E. R. A DensityFunctional Model of the Dispersion Interaction. J. Chem. Phys. 2005, 123 (15), 154101.

(61) Grimme, S. Semiempirical GGA-Type Density Functional Constructed with a Long-Range Dispersion Correction. J. Comput. Chem. 2006, 27 (15), 1787-1799.

(62) Tkatchenko, A.; Scheffler, M. Accurate Molecular Van Der Waals Interactions from Ground-State Electron Density and FreeAtom Reference Data. Phys. Rev. Lett. 2009, 102 (7), 073005.

(63) Grimme, S.; Antony, J.; Ehrlich, S.; Krieg, H. $\mathrm{A}$ Consistent and Accurate $\mathrm{Ab}$ Initio Parametrization of Density Functional Dispersion Correction (DFT-D) for the 94 Elements H-Pu. J. Chem. Phys. 2010, 132 (15), 154104.

(64) Vydrov, O. A.; van Voorhis, T. Nonlocal van Der Waals Density Functional: The Simpler the Better. J. Chem. Phys. 2010, 133 (24), 244103.

(65) Caldeweyher, E.; Ehlert, S.; Hansen, A.; Neugebauer, H.; Spicher, S.; Bannwarth, C.; Grimme, S. A Generally Applicable AtomicCharge Dependent London Dispersion Correction. J. Chem. Phys. 2019, 150 (15), 154122.

(66) Mardirossian, N.; Head-Gordon, M. Mapping the Genome of Meta-Generalized Gradient Approximation Density Functionals: The Search for B97M-V. J. Chem. Phys. 2015, 142 (7), 074111-32.

(67) Mardirossian, N.; Head-Gordon, M. $\omega$ B97MV: A Combinatorially Optimized, RangeSeparated Hybrid, Meta-GGA Density Functional with VV10 Nonlocal Correlation. J. Chem. Phys. 2016, 144 (21), 214110.

(68) Sun, J.; Ruzsinszky, A.; Perdew, J. P. Strongly Constrained and Appropriately Normed Semilocal Density Functional. Phys. Rev. Lett. 2015, 115 (3), 036402.

(69) Becke, A. D. Real-Space Post-Hartree-Fock Correlation Models. J. Chem. Phys. 2005, 122 (6), 064101.

(70) Peverati, R.; Truhlar, D. G. M11-L: A Local Density Functional That Provides Improved Accuracy for Electronic Structure 
Calculations in Chemistry and Physics. J. Phys. Chem. Lett. 2012, 3, 117-124.

(71) Janesko, B. G. Rung 3.5 Density Functionals: Another Step on Jacob's Ladder. Int. J. QUANTUM Chem. 2012, 113 (2), 83-88.

(72) Becke, A. D. Density Functionals for Static, Dynamical, and Strong Correlation. J. Chem. Phys. 2013, 138, 074109.

(73) Kong, J.; Proynov, E. Density Functional Model for Nondynamic and Strong Correlation. J. Chem. Theory Comput. 2016, 12 (1), 133-143.

(74) Verma, P.; Truhlar, D. G. Can Kohn-Sham Density Functional Theory Predict Accurate Charge Distributions for Both SingleReference and Multi-Reference Molecules? Phys. Chem. Chem. Phys. 2017, 19 (20), 12898-12912.

(75) Becke, A. D. Density-Functional ExchangeEnergy Approximation with Correct Asymptotic-Behavior. Phys. Rev. A 1988, 38, 3098-3100.

(76) Lee, C.; Yang, W.; Parr, R. G. Development of the Colle-Salvetti Correlation-Energy Formula Into a Functional of the ElectronDensity. Phys. Rev. B 1988, 37, 785-789.

(77) Becke, A. D. Density-Functional Thermochemistry. 3. The Role of Exact Exchange. J. Chem. Phys. 1993, 98 (7), 5648-5652.

(78) Stephens, P.; Devlin, F.; Chabalowski, C.; Frisch, M. J. Ab-Initio Calculation of Vibrational Absorption and CircularDichroism Spectra Using DensityFunctional Force-Fields. J. Phys. Chem. 1994, 98 (45), 11623-11627.

(79) Shao, Y.; Gan, Z.; Epifanovsky, E.; Gilbert, A. T. B.; Wormit, M.; Kussmann, J.; Lange, A. W.; Behn, A.; Deng, J.; Feng, X.; et al. Advances in Molecular Quantum Chemistry Contained in the Q-Chem 4 Program Package. Mol. Phys. 2015, 113 (2), 184-215.

(80) Gilbert, A. T. B. IQmol; 2019.

(81) Perdew, J. P.; Schmidt, K. Jacob's Ladder of Density Functional Approximations for the Exchange-Correlation Energy. AIP Conf. Proc. 2001, 577, 1-20.

(82) Peverati, R.; Truhlar, D. G. Quest for a Universal Density Functional: The Accuracy of Density Functionals across a Broad Spectrum of Databases in Chemistry and Physics. Philos. Trans. R. Soc. Lond. Ser. Math. Phys. Eng. Sci. 2014, 372 (2011), 20120476.
(83) Yu, H. S.; Li, S. L.; Truhlar, D. G. Perspective: Kohn-Sham Density Functional Theory Descending a Staircase. J. Chem. Phys. 2016, 145 (13), 130901-24.

(84) Becke, A. D. Perspective: Fifty Years of Density-Functional Theory in Chemical Physics. J. Chem. Phys. 2014, 140 (18), 18A301-19.

(85) Rappoport, D.; Crawford, N. R. M.; Furche, F.; Burke, K. Approximate Density Functionals: Which Should I Choose? In Encyclopedia of Inorganic and Bioinorganic Chemistry; Scott, R. A., Ed.; John Wiley \& Sons, Ltd: Chichester, UK, 2011; p eibc0380.

(86) Goerigk, L.; Mehta, N. A Trip to the Density Functional Theory Zoo: Warnings and Recommendations for the User. Aust. J. Chem. 2019, 72 (8), 563.

(87) Davidson, E. R.; Feller, D. Basis Set Selection for Molecular Calculations. Chem. Rev. 1986, 86 (4), 681-696.

(88) Balabin, R. M. Communications: Intramolecular Basis Set Superposition Error as a Measure of Basis Set Incompleteness: Can One Reach the Basis Set Limit without Extrapolation? J. Chem. Phys. 2010, 132 (21), 211103.

(89) Sure, R.; Grimme, S. Corrected Small Basis Set Hartree-Fock Method for Large Systems. 2013, 34 (19), 1672-1685.

(90) van Duijneveldt, F. B.; van Duijneveldt-van de Rijdt, J. G. C. M.; van Lenthe, J. H. State of the Art in Counterpoise Theory. Chem. Rev. 1994, 94 (7), 1873-1885.

(91) Ditchfield, R.; Hehre, W. J.; Pople, J. A. SelfConsistent Molecular-Orbital Methods. IX. An Extended Gaussian-Type Basis for Molecular-Orbital Studies of Organic Molecules. J. Chem. Phys. 1971, 54 (2), 724728.

(92) Hariharan, P. C.; Pople, J. A. The Influence of Polarization Functions on Molecular Orbital Hydrogenation Energies. Theor. Chim. Acta 1973, 28 (3), 213-222.

(93) Dill, J. D.; Pople, J. A. Self-consistent Molecular Orbital Methods. XV. Extended Gaussian-type Basis Sets for Lithium, Beryllium, and Boron. J. Chem. Phys. 1975, 62 (7), 2921-2923.

(94) Binkley, J. S.; Pople, J. A. Self-consistent Molecular Orbital Methods. XIX. Splitvalence Gaussian-type Basis Sets for Beryllium. J. Chem. Phys. 1977, 66 (2), 879880. 
(95) Francl, M. M.; Pietro, W. J.; Hehre, W. J.; Binkley, J. S.; Gordon, M. S.; DeFrees, D. J.; Pople, J. A. Self-Consistent Molecular Orbital Methods. XXIII. A Polarization-Type Basis Set for Second-Row Elements. $J$. Chem. Phys. 1982, 77 (7), 3654-3665.

(96) Gordon, M. S.; Binkley, J. S.; Pople, J. A.; Pietro, W. J.; Hehre, W. J. Self-Consistent Molecular-Orbital Methods. 22. Small SplitValence Basis Sets for Second-Row Elements. J. Am. Chem. Soc. 1982, 104 (10), 2797-2803.

(97) Rassolov, V. A.; Pople, J. A.; Ratner, M. A.; Windus, T. L. 6-31G * Basis Set for Atoms K through Zn. J. Chem. Phys. 1998, 109 (4), 1223-1229.

(98) Rassolov, V. A.; Ratner, M. A.; Pople, J. A.; Redfern, P. C.; Curtiss, L. A. 6-31G* Basis Set for Third-Row Atoms. J. Comput. Chem. 2001, 22 (9), 976-984.

(99) Witte, J.; Neaton, J. B.; Head-Gordon, M. Push It to the Limit: Characterizing the Convergence of Common Sequences of Basis Sets for Intermolecular Interactions as Described by Density Functional Theory. $J$. Chem. Phys. 2016, 144 (19), 194306.

(100) Dunning, T. H. Gaussian-Basis Sets for Use in Correlated Molecular Calculations. 1. The Atoms Boron Through Neon and Hydrogen. J. Chem. Phys. 1989, 90 (2), 1007-1023.

(101) Woon, D. E.; Dunning Jr., T. H. Gaussian Basis Sets for Use in Correlated Molecular Calculations. III. The Atoms Aluminum through Argon. J. Chem. Phys. 1993, 98 (2), 1358-1371.

(102) Woon, D. E.; Dunning, T. H. Gaussian Basis Sets for Use in Correlated Molecular Calculations. IV. Calculation of Static Electrical Response Properties. J. Chem. Phys. 1994, 100 (4), 2975-2988.

(103) Wilson, A. K.; Woon, D. E.; Peterson, K. A.; Dunning, T. H. Gaussian Basis Sets for Use in Correlated Molecular Calculations. IX. The Atoms Gallium through Krypton. J. Chem. Phys. 1999, 110 (16), 7667-7676.

(104) Papajak, E.; Leverentz, H. R.; Zheng, J.; Truhlar, D. G. Efficient Diffuse Basis Sets: Cc-PV $\times \mathrm{Z}+$ and Maug-Cc-PV $\propto Z$. J. Chem. Theory Comput. 2009, 5 (5), 1197-1202.

(105) Weigend, F.; Ahlrichs, R. Balanced Basis Sets of Split Valence, Triple Zeta Valence and Quadruple Zeta Valence Quality for $\mathrm{H}$ to Rn: Design and Assessment of Accuracy.
Phys. Chem. Chem. Phys. 2005, 7 (18), 3297-3305.

(106) Jensen, F. Polarization Consistent Basis Sets. 4: The Elements $\mathrm{He}, \mathrm{Li}, \mathrm{Be}, \mathrm{B}, \mathrm{Ne}, \mathrm{Na}$, $\mathrm{Mg}, \mathrm{Al}$, and Ar †. J. Phys. Chem. A 2007, 111 (44), 11198-11204.

(107) Jensen, F. Polarization Consistent Basis Sets: Principles. J. Chem. Phys. 2001, 115 (20), 9113-9125.

(108) Jensen, F.; Helgaker, T. Polarization Consistent Basis Sets. V. The Elements SiCl. J. Chem. Phys. 2004, 121 (8), 34633470 .

(109) Jensen, F. Polarization Consistent Basis Sets. VII. The Elements K, Ca, Ga, Ge, As, $\mathrm{Se}, \mathrm{Br}$, and Kr. J. Chem. Phys. 2012, 136 (11), 114107.

(110) Kruse, H.; Goerigk, L.; Grimme, S. Why the Standard B3LYP/6-31G* Model Chemistry Should Not Be Used in DFT Calculations of Molecular Thermochemistry: Understanding and Correcting the Problem. J. Org. Chem. 2012, 77 (23), 10824-10834.

(111) Yepes, D.; Valenzuela, J.; Martínez-Araya, J. I.; Pérez, P.; Jaque, P. Effect of the Exchange-Correlation Functional on the Synchronicity/Nonsynchronicity in Bond Formation in Diels-Alder Reactions: A Reaction Force Constant Analysis. Phys. Chem. Chem. Phys. 2019, 21 (14), 74127428.

(112) Migliore, A. How To Extract Quantitative Information on Electronic Transitions from the Density Functional Theory "Black Box." J. Chem. Theory Comput. 2019, 15 (9), 4915-4923.

(113) Caricato, M.; Trucks, G. W.; Frisch, M. J.; Wiberg, K. B. Electronic Transition Energies: A Study of the Performance of a Large Range of Single Reference Density Functional and Wave Function Methods on Valence and Rydberg States Compared to Experiment. J. Chem. Theory Comput. 2010, 6 (2), 370-383.

(114) Isegawa, M.; Peverati, R.; Truhlar, D. G. Performance of Recent and HighPerformance Approximate Density Functionals for Time-Dependent Density Functional Theory Calculations of Valence and Rydberg Electronic Transition Energies. J. Chem. Phys. 2012, 137, 244104.

(115) Hein, S. M.; Kopitzke, R. W.; Nalli, T. W.; Esselman, B. J.; Hill, N. J. Use of ${ }^{1} \mathrm{H},{ }^{13} \mathrm{C}$, and 19 F-NMR Spectroscopy and Computational Modeling To Explore Chemoselectivity in the Formation of a 
Grignard Reagent. J. Chem. Educ. 2015, 92 (3), 548-552.

(116) Wheeler, S. E.; Houk, K. N. Integration Grid Errors for Meta-GGA-Predicted Reaction Energies: Origin of Grid Errors for the M06 Suite of Functionals. J. Chem. Theory Comput. 2009, 6 (2), 395-404.

(117) Mardirossian, N.; Head-Gordon, M. Characterizing and Understanding the Remarkably Slow Basis Set Convergence of Several Minnesota Density Functionals for Intermolecular Interaction Energies. $J$. Chem. Theory Comput. 2013, 9 (10), 44534461.

(118) Mardirossian, N.; Head-Gordon, M. How Accurate Are the Minnesota Density Functionals for Noncovalent Interactions, Isomerization Energies, Thermochemistry, and Barrier Heights Involving Molecules $\backslash$ ldots. J. Chem. Theory Comput. 2016, 12 (9), 4303-4325.

(119) Bootsma, A. N.; Wheeler, S. E. Popular Integration Grids Can Result in Large Errors in DFT-Computed Free Energies. 20.

(120) Seeger, R.; Pople, J. A. Self-consistent Molecular Orbital Methods. XVIII. Constraints and Stability in Hartree-Fock Theory. J. Chem. Phys. 1977, 66 (7), 30453050.

(121) Bauernschmitt, R.; Ahlrichs, R. Stability Analysis for Solutions of the Closed Shell Kohn-Sham Equation. J. Chem. Phys. 1996, 104 (22), 9047-9052.

(122) Cramer, C. J. Essentials of Computational Chemistry: Theories and Models; 2013.

(123) Mulliken, R. S. Electronic Population Analysis on LCAO-MO Molecular Wave Functions. 1. J. Chem. Phys. 1954, 23 (10), 1833-1840.

(124) Mulliken, R. S. Electronic Population Analysis on LCAO-MO Molecular Wave Functions. 2. Overlap Populations, Bond Orders, and Covalent Bond Energies. J. Chem. Phys. 1954, 23 (10), 1841-1846.

(125) Mulliken, R. S. Electronic Population Analysis on LCAO-MO Molecular Wave Functions. 3. Effects of Hybridization on Overlap and Gross AO Populations. J. Chem. Phys. 1955, 23 (12), 2338-2342.

(126) Mulliken, R. S. Electronic Population Analysis on LCAO-MO Molecular Wave Functions. 4. Bonding and Antibonding in Lcao and Valence-Bond Theories. J. Chem. Phys. 1954, 23 (12), 2343-2346.
(127) Chirlian, L. E.; Francl, M. M. Atomic Charges Derived from Electrostatic Potentials: A Detailed Study. J. Comput. Chem. 1987, 8 (6), 894-905.

(128) Breneman, C. M.; Wiberg, K. B. Determining Atom-Centered Monopoles from Molecular Electrostatic Potentials. The Need for High Sampling Density in Formamide Conformational Analysis. $J$. Comput. Chem. 1990, 11 (3), 361-373.

(129) Ostrovsky, V. N. Towards a Philosophy of Approximations in the 'Exact' Sciences. HYLE - Int. J. Philos. Chem. 2005, 11, 101126.

(130) Pham, B. Q.; Gordon, M. S. Can Orbitals Really Be Observed in Scanning Tunneling Microscopy Experiments? J. Phys. Chem. A 2017, 121 (26), 4851-4852.

(131) Truhlar, D. G.; Hiberty, P. C.; Shaik, S.; Gordon, M. S.; Danovich, D. Orbitals and the Interpretation of Photoelectron Spectroscopy and (e,2e) Ionization Experiments. Angew. Chem. Int. Ed. 2019, 58 (36), 12332-12338.

(132) Scerri, E. R. Have Orbitals Really Been Observed? Sci. Educ. 3.

(133) Boys, S. F. Construction of Some Molecular Orbitals to Be Approximately Invariant for Changes from One Molecule to Another. Rev. Mod. Phys. 1960, 32 (2), 296299.

(134) Lennard-Jones, J.; Pople, J. A. A Survey of the Principles Determining the Structure and Properties of Molecules. Part 1.-The Factors Responsible for Molecular Shape and Bond Energies. Discuss Faraday Soc 1951, $10(0), 9-18$.

(135) Edmiston, C.; Ruedenberg, K. Localized Atomic and Molecular Orbitals. Rev. Mod. Phys. 1963, 35 (3), 457-464.

(136) Edmiston, C.; Ruedenberglb, K. Chemical Binding in the Water Molecule". J. Phys. Chem. 26.

(137) Roos, B. O.; Lindh, R.; Malmqvist, P. Å.; Veryazov, V.; Widmark, P.-O. Multiconfigurational Quantum Chemistry; Wiley: Hoboken, New Jersey, 2016. 